\title{
LA FUSIÓN GLACIAR EN EL PIRINEO: CONDICIONANTES AMBIENTALES Y PRIMEROS RESULTADOS BIANUALES EN EL GLACIAR DEL INFIERNO (PIRINEO ARAGONÉS)
}

\author{
L. Cancer Pomar (1), F. Lampre Vitaller (2),
} A. Ollero Ojeda (3) y J. Del Valle Melendo (4)

(1) Área de Geografía Física, Universidad de Jaén

(2)Consejo de Protección de los Glaciares del Pirineo Aragonés

(3) Area de Geografía Física, Universidad del País Vasco

(4) Dpto. de Geografía y Ordenación del Territorio, Universidad de Zaragoza

Resumen: Se presentan datos acerca de las características ambientales del Glaciar del Infierno y de la regresión sufrida en el último siglo, así como los valores de los caudales de fusión de dos ciclos diarios en este glaciar, medidos a finales de dos veranos consecutivos (años 1998 y 1999). La relación entre este dato y los ritmos térmicos es muy estrecha, lo cual permite disponer de un dato más, de gran importancia, para explicar el control ambiental en los sistemas de drenaje de la fusión glaciar, así como la acusada regresión glaciar de las últimas décadas, indicativa de balances entre la acumulación nival y la ablación claramente negativos. Los datos se acompañan de medidas de retroceso glaciar entre los veranos de 1998 y 1999.

Palabras clave: regresión glaciar, caudales de fusión, ciclo diario, glaciares pirenaicos.

\begin{abstract}
Data are presented about the environmental characters of the Infiernos glacier and of the regression suffered in the last century, as well as the meltwater flow values corresponding to two daily cycle of the Infierno glacier. These values were measured at the end of the summer of 1998 and 1999. There is a close relationship between these data and both thermic rythms. This fact suggests that meltwater flow should become a useful additional parameter to explain the environmental control of the drainage systems derived from the glacier fusion, as well as the serious glacier regression happened in the last decades, indicative of balances between the accumulation nival and the ablation clearly negative. The data are accompanied of measures of regression glacier among the summers of 1998 and 1999.
\end{abstract}

Key words: glacier regression, meltwater flow, daily cycle, Pyrenean glaciers. 


\section{Introducción}

El estudio de la fusión glaciar en el Pirineo no ha sido tan apenas abordado hasta la fecha. A diferencia de lo que ocurre en otras cordilleras glaciadas (como en los Alpes), en las que se vienen realizando periódicamente campañas de este fenómeno que sirven, entre otros objetivos, para establecer los balances glaciares, los estudios glaciológicos pirenaicos se han centrado, desde finales del siglo xIX, en los catálogos de masas glaciares y, en algunos casos, en la cartografía geomorfológica de su entorno. Esta carencia de información llevó a los autores de este artículo a plantearse la toma de datos sistemática del proceso de fusión en varios glaciares pirenaicos a lo largo de una serie de años sucesivos. Los primeros resultados, referidos a los glaciares de Infiernos y Maladeta (Lampre et al., 1999; Ollero et al., 2000; Cancer et al., 2001 y en prensa) arrojan interesantes resultados de los ritmos diarios de fusión. En el presente artículo comparamos, para el glaciar de Infiernos, los datos de las dos primeras campañas (1998 y 1999), relacionándolos con los factores ambientales que en mayor medida determinan la fusión glaciar. También se explica brevemente el estado de la cuestión y el método de estudio aplicado a la obtención de los datos. Finalmente, los volúmenes de fusión -mayores en la campaña de 1999 que en la del año anteriorpermiten entender los acusados valores de retroceso de la masa de hielo, tanto en extensión como en potencia.

\section{2. Área de estudio}

A finales del siglo xx los Pirineos conservan 570 ha de superficie glaciar. De ellas, 342 pertenecen al sector español (según nuestros trabajos de campo de inicios de septiembre de 2000). Se reparten entre trece glaciares (algunos de infimo tamaño, a punto de convertirse en simples heleros) y varios heleros ubicados sobre el territorio septentrional de la provincia de Huesca, en macizos que superan los $3.000 \mathrm{~m}$ de altitud y, en su mayoría, en exposiciones favorables ( $N$ y NE). Se trata de masas de hielo ubicadas en circos o plateaux al pie de afiladas crestas rocosas, con cotas inferiores a partir de unos $2.700 \mathrm{~m}$. Se distribuyen a lo largo de $90 \mathrm{~km}$ aproximadamente entre los macizos de Balaitús o Moros (glaciado en su vertiente norte o francesa), en el límite oeste (alta cuenca del río Gállego), y de La Maladeta, en el este (cabeceras de los ríos Ésera y Noguera Ribagorzana). La localización precisa de estos aparatos puede consultarse en Martínez de Pisón y Arenillas (1988), Chueca y Lampre (1994) o Chueca et al. (1998). Mientras, en la vertiente francesa únicamente subsisten 228 ha en la misma fecha (René, 2001). 
La elección de los aparatos glaciares sobre los que llevar a cabo el estudio del ritmo diario de los caudales de fusión ha estado condicionada fundamentalmente por su morfología. Tal como explicamos en Lampre et al. (1999), las formas en embudo son las más apropiadas, pues las escorrentías supra y subglaciar se concentran en el frente terminal del hielo, dando lugar a un único colector de las aguas de fusión. Esto es lo que ocurre en el glaciar de Infiernos, ubicado en la vertiente norte del macizo de idéntica denominación, en pleno Pirineo Axial aragonés, en la cabecera de la cuenca del Caldarés, afluente por la izquierda del río Gállego (figura 1).

Este aparato glaciar se halla enmarcado por los picos del Infierno o los Infiernos llamado también Quijada de Pondiellos, los cuales se individualizan en tres cimas principales, alcanzando los $3.082 \mathrm{~m}$ en su punto culminante (Infierno Central). Están constituidos por pelitas y calizas devónicas atravesadas por potentes franjas de mármoles. Estos picos conforman una cresta de eje NO-SE, lo cual permite una orientación apropiada para el desarrollo glaciar en su vertiente NE, favorecida además por lo abrupto del sector, que explica la existencia de extensas umbrías. La excavación de los circos glaciares se ha visto facilitada por las características estructurales de la zona, pues coinciden con fallas de sentido N-S. Los caudales de fusión alimentan los ibones (término aplicado en Aragón a los lagos de origen glaciar) Azules, ubicados al E-NE de las masas de hielo (Cancer, 1995). En todo el sector se suceden episodios glaciares ordenados en el tiempo, desde las evidencias de morrenas tardiglaciares hasta las de la Pequeña Edad del Hielo (Serrano y Agudo, 1988). Las características geomorfológicas de la zona aparecen cartografiadas en la figura 1.

Según la bibliografía glaciológica referida a este sector, en el macizo de los Infiernos se ha señalado tradicionalmente la existencia de dos glaciares (occidental y oriental) y un helero, en circos de orientación N-NE (Martínez de Pisón y Arenillas, 1988; Serrano y Agudo, 1988; Arenillas et al., 1992; Chueca y Lampre, 1994; Chueca et al., 1998). Pero algunos trabajos ya indicaban la evidente evolución regresiva del aparato oriental: el aparato occidental es el más activo (..); el glaciar oriental es menos dinámico, evoluciona hacia un belero estático (Chueca y Lampre, 1994, p. 21). En nuestros primeros trabajos de campo, de septiembre de 1998, pudimos comprobar que la categoría de helero ya había sido alcanzada por este anterior glaciar, de manera que en el macizo de Infiernos sólo queda actualmente un aparato realmente glaciar, el denominado tradicionalmente glaciar occidental, con una extensión de 9,6 ha, entre las cotas $2676 \mathrm{~m}$ y $2900 \mathrm{~m}$ (datos medidos in situ el 11 de septiembre de 2000). Su relativamente baja cota inferior, en comparación con las restantes masas glaciares surpirenaicas, puede ser debida a la entidad de la umbría sobre la que se desarrolla, al pie de la imponente vertiente septentrional de los Infiernos, o a su posición occidental en el territorio glaciado pirenaico, favorecedora de las advecciones atlánticas O-NO (origen de buena parte de las precipitaciones del sector). La fuerte pendiente de la rampa de hielo facilita la existencia de abundantes grietas de tracción en su tercio superior, otorgando a este glaciar una morfología vigorosa que se ve magnificada por su imponente marco estructural. 
MAPA GEOMORFOLÓGICO DEL MACIZO DEL INFIERNO LEYENDA

MODELADO GLACIAR
GLACIARISMO FUNCIONAL
Glaciar
Forieros
Aristas de divisoria entre los
circos glaciares
Hoom o pico resultante del
recortamiento glaciar
Brectias o collados en las anistas
divisorias

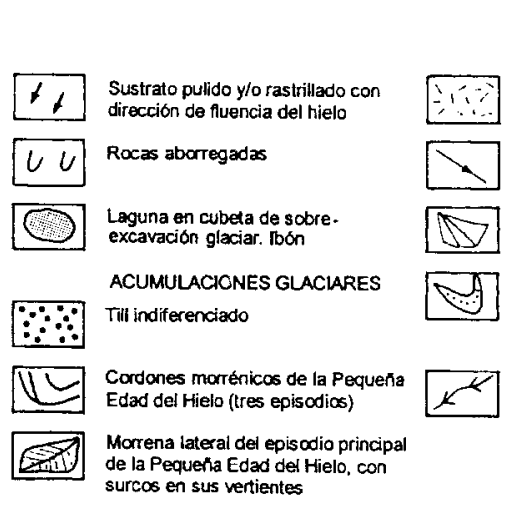

MODELADO PERIGLACIAR YNIVAL

Canchaies y taludes de derrubios

Canales de aludes

Conos de derrubios y/o aludes

Morrena de nevero

MOQELADQ HIORICO

Baranco de incisión linea (aguas de fusión glaciar)

lequena Edad del Hielo, con

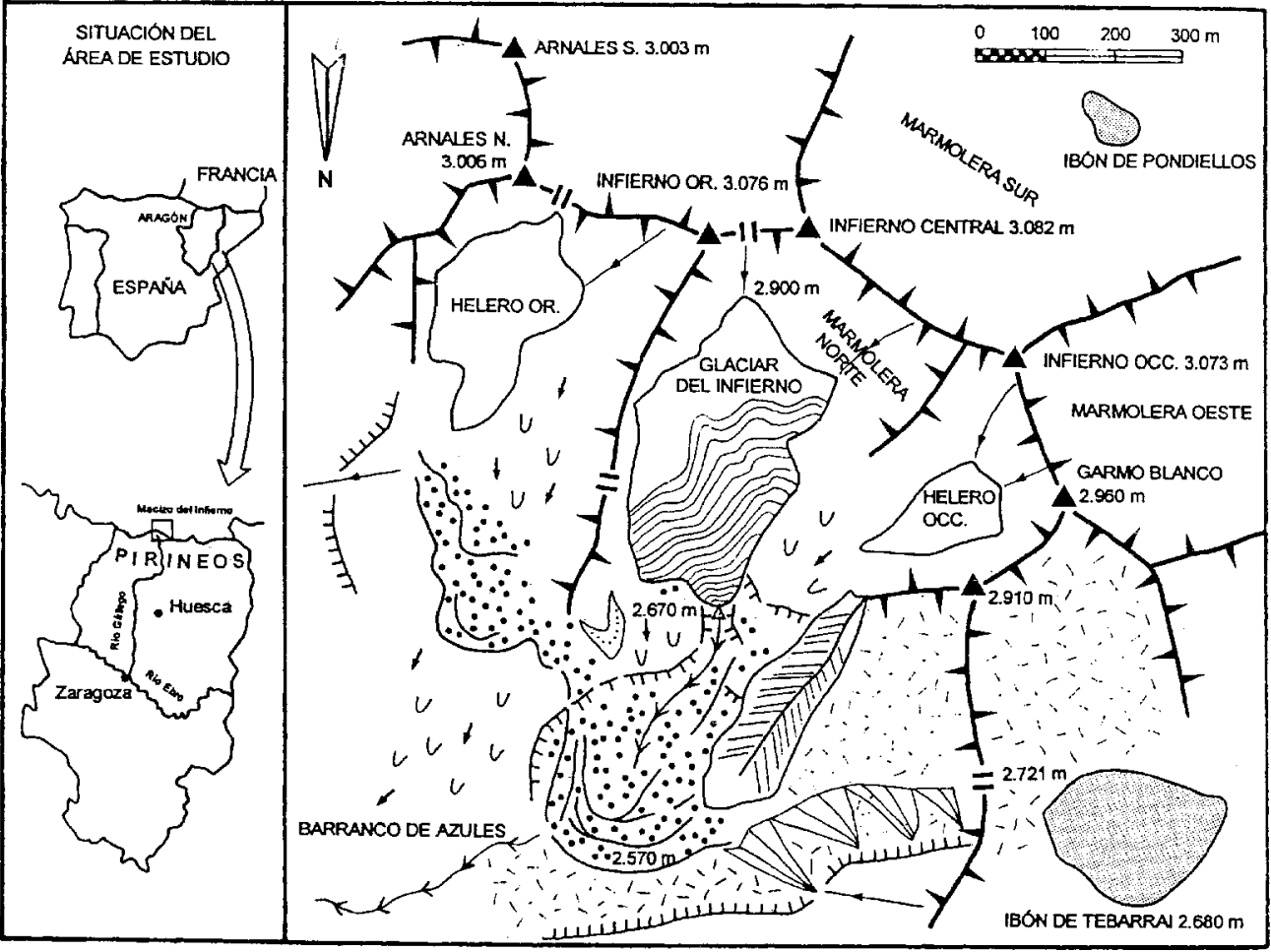

$\Delta$ Punto de medición de los caudales de fusión

Figura 1. Situación del Glaciar del Infierno y mapa geomorfológico del área de estudio 


\section{Estado de la cuestión y planteamiento del trabajo}

En el Pirineo aragonés persisten actualmente los últimos glaciares blancos de la Península Ibérica. Se trata de masas sujetas a un rápido retroceso desde finales de la Pequeña Edad del Hielo, especialmente marcado en las dos últimas décadas. El balance glaciar resulta claramente negativo. Diferentes trabajos ponen de manifiesto las causas climáticas explicativas del proceso (Lampre, 1998; Arenillas et al., 2000). Cabe indicar, en este sentido, una carencia con la que topan los estudios glaciológicos de la cordillera: la falta de estaciones meteorológicas de altitud, de manera que no se dispone de datos climáticos directos de los ámbitos glaciares, lo cual impide, por ejemplo, disponer de valores de acumulación nival, fundamentales en glaciología.

Dada esta inexistencia de datos directos de entrada de nieve en la masa glaciar, nos hemos aproximado al estudio del balance glaciar estudiando su otro componente: las salidas de agua. Los problemas que a este respecto provocan los caudales procedentes de la fusión de la nieve que recubre el cuerpo de hielo y que se mezclan los derivados de ablación directa de éste obligan a realizar los trabajos de campo en un corto periodo de tiempo, a finales del verano, en el que la cobertura nival es mínima y los glaciares se hallan descubiertos -en nuestras latitudes- en casi toda su extensión (Lampre et al., 1999). De esta manera, en esas escasas fechas, se registran datos de fusión directa del hielo en su mayor parte. Cabe indicar que mientras durante el invierno los caudales de fusión glaciar son originados mayoritariamente por el flujo basal, los caudales estivales proceden en su mayoría de la fusión superficial (Sugden y John, 1976), fenómeno que por sí solo justifica la elección de fechas de medición en que la cobertura nival desvirtúe los datos lo menos posible.

Los ritmos de desagüe del agua de fusión glaciar han sido bien estudiados en los Alpes, no así en los Pirineos. La relación diaria variación térmica-caudales de fusión es muy estrecha, aunque no totalmente sincrónica, ya que la elevación de temperaturas antecede en varias horas al aumento del flujo. También resulta interesante conocer el papel del calor estacional acumulado en el cuerpo glaciar, pues según el seguimiento de los glaciares alpinos la amplitud de la fluctuación tiende a acentuarse en el transcurso del verano. Por otra parte, la fusión diaria sigue un ritmo que anticipa progresivamente la hora del pico de descarga máxima, como ya hace casi tres décadas comprobaron Vivian y Zumstein (1973) en el glaciar de l'Argentière (Mont Blanc). Mientras que el pico máximo se obtiene hacia las 23-24 horas al comienzo del verano, se adelanta hasta las 16-17 horas en septiembre ${ }^{1}$. Este adelanto paulatino se relaciona con la progresiva incisión estival de la red de drenaje supraglaciar, así como con el desarrollo y apertura de las cavidades y túneles endo y subglaciares, con lo que se va ganando progresivamente en eficacia y rapidez de desagüe. Por el contrario, el invierno recongela cualquier estructura del sistema de drenaje, a excepción de la base del hielo. En cualquier caso, factores ajenos a la estricta fusión, como una nevada estival (reduciendo momentáneamente la 
ablación) o una tormenta (aumentando la descarga), pueden causar bruscas modificaciones de estos ritmos "normales" diarios.

A principios de septiembre de los años 1998 y 1999, el tercio inferior del glaciar de Infiernos se halla surcado por numerosos canales de fusión (bédières) meandriformes de baja sinuosidad. Llama la atención el hecho de que éstos son sensiblemente más numerosos y desarrollados en 1999 que en el año anterior, confirmando la tendencia regresiva del glaciar. Mientras que en 1998 sus medidas eran de 1-2 m de profundidad y 0,5-1 m de ancho, en 1999 pueden llegar fácilmente a 2,5 m de profundidad y 1,5 $\mathrm{m}$. de ancho. Estos bedières constituyen una red subparalela y convergente en el frente del glaciar que encauza las aguas de fusión supraglaciar. El reducido espesor (inferior a $5 \mathrm{~m}$ ) y escaso dinamismo del aparato glaciar en su sector inferior, limitado a una masa de hielo inactiva y terminal, no induce a la pérdida de las aguas superficiales en sumideros (no existen moulins) o grietas (crevasses), inexistentes en las observaciones realizadas durante las fechas de nuestro estudio. De esta forma, regueros y bédières confluyen sin obstáculos reseñables en el frente del glaciar y en el embudo rocoso que se origina inmediatamente a sus pies, canalizando prácticamente la totalidad de las aguas supraglaciares. En este punto también surgen las aguas de fusión subglaciar y endoglaciar, cuya observación y análisis comporta serias dificultades.

\section{Objetivos y metodología}

La suma de los caudales aportados por todas las aguas de fusión del glaciar del Infierno y su ritmo diario de descarga constituyen los principales objetivos de este estudio. El método de medición de caudales aplicado se expone en Lampre et al. (1999) y Cancer et al. (2001 y en prensa). En las líneas siguientes explicamos de manera comparada los resultados obtenidos en las campañas de 1998 y 1999.

Los trabajos de campo se llevaron a cabo los días 4-5 de septiembre de 1988 y 10-11 de septiembre de 1999. Para la medición se eligió el extremo inferior de la lengua de hielo, del que surge una corriente de fusión sobre un cauce de fuerte pendiente que se encaja entre la roca enmarcante del glaciar (orilla derecha) y el depósito morrénico (orilla izquierda), a $2.670 \mathrm{~m}$ de altitud. A escasos metros al pie del extremo de la lengua del hielo, el cauce pierde pendiente en un punto muy concreto donde la comiente se remansa un tanto. Alli se efectuaron las medidas, acondicionando con los propios bloques del cauce una pequeña represa que controlaba la mayor parte del caudal (ver la localización en la figura 1). Este punto, situado a sólo $3 \mathrm{~m}$ del extremo final de la lengua de hielo en 1998, distaba 11,5 m en 1999, debido a la regresión glaciar experimentada.

Respecto a la selección del punto de medición, conviene indicar que los trabajos de campo que hemos efectuado sincrónicamente en otros aparatos glaciares pirenai- 
cos nos permiten comprobar importantes diferencias en los ritmos diarios de variaciones de caudal debidas, además de al propio comportamiento diferenciado de los distintos cuerpos de hielo, a la ubicación concreta de los lugares en los que se efectúan las mediciones, que pueden encontrarse inmediatamente al pie del hielo o a cierta distancia, separados de aquél por depósitos morrénicos de otro tipo que actúan a modo de esponja y amortiguan las curvas de variación (Ollero et al., 2000). No es éste el caso de nuestras mediciones en el Glaciar de Infiernos, efectuadas, como acabamos de ver, inmediatamente al pie del hielo.

Las mediciones de la velocidad de la corriente se realizaron con un molinete o correntímetro electrónico (Ott C2), e iban acompañadas de mediciones paralelas del perímetro mojado del punto de muestreo. Se trató de realizar el mayor número posible de medidas a lo largo de 24 horas: una medición cada hora entre las $8 \mathrm{~h}$. y las $22 \mathrm{~h}$., y una medición cada dos horas entre las $22 \mathrm{~h}$. y las $8 \mathrm{~h}^{2}$. Esta diferente frecuencia de medidas diurnas y nocturnas se justifica sobre todo en que los ritmos de variaciones de caudal son mucho menores por la noche que por el día. Además, las dificultades propias del trabajo en alta montaña (Bocquet et Thomas, 1989), se acrecientan en horario nocturno. Junto a los valores de caudal, en cada medición se anotaron los factores ambientales que más influyen en el proceso de fusión: temperatura del aire y del hielo, nubosidad, viento y porcentaje de la superficie glaciar con insolación.

\section{Resultados}

Los resultados obtenidos en ambos años demuestran que existe un régimen diario de fusión del hielo en el glaciar del Infierno con unos valores muy contrastados, indicativos de un ritmo similar al observado en otros glaciares de montaña en latitudes medias.

Comenzamos el análisis con los datos referidos a la fusión glaciar en 1998 (4-5 septiembre). Las figuras 2,3 y 4 , junto a la tabla 1 , sirven de apoyo gráfico y numérico a estas explicaciones.

- El caudal mínimo se registró a las 8:14 horas del 5 de septiembre, con 1,25 l/s, y el máximo a las 16:12 horas, con $105,51 \mathrm{l} / \mathrm{s}$. Es decir, la fusión glaciar llegó a alcanzar durante el día un valor 84,4 veces superior al de la noche precedente.

- El descenso de caudal en la tarde del día 5 fue extrañamente rápido en comparación con la tarde del día 4. Posiblemente se deba al decremento térmico brusco entre las 15:05 h y las 16:12 h, con una curva de descenso mucho más pronunciada que la de la tarde-noche del día anterior. 
- Los resultados de caudal pueden cotejarse con los dos elementos climáticos que más parecen controlar la fusión glaciar: la temperatura y la insolación. La temperatura del hielo, medida a $5 \mathrm{~cm}$ de profundidad, no varía de forma perceptible a lo largo de las 24 horas, situándose siempre en cero grados. La temperatura ambiental presenta una curva que refleja una clara influencia en el caudal. Además, el incremento o decremento hídrico es posterior al térmico en unas 2 horas, lo cual viene a confirmar la clara relación causa-efecto entre ambos factores, y a raíz del descenso nocturno se produce un decremento de caudal que alcanza su valor mínimo a las $8: 14 \mathrm{~h}(1,25 \mathrm{l} / \mathrm{s})$. A las $10: 38 \mathrm{~h}$, cuando la insolación sobre el glaciar comienza a ser apreciable y la temperatura ha subido de forma importante, el caudal es todavía muy bajo $(1,54 \mathrm{l} / \mathrm{s})$. Hay una curiosa ralentización del incremento de caudal entre las 14:10 h y las 15:05 h que puede ser la respuesta al descenso térmico que se había detectado entre las $12 \mathrm{~h}$ y las $13 \mathrm{~h}$.

- Podemos destacar el hecho de que el día 4 presentó unos valores más altos de caudal que el día 5, aun cuando no hubo precipitación acuosa ni lució el sol.

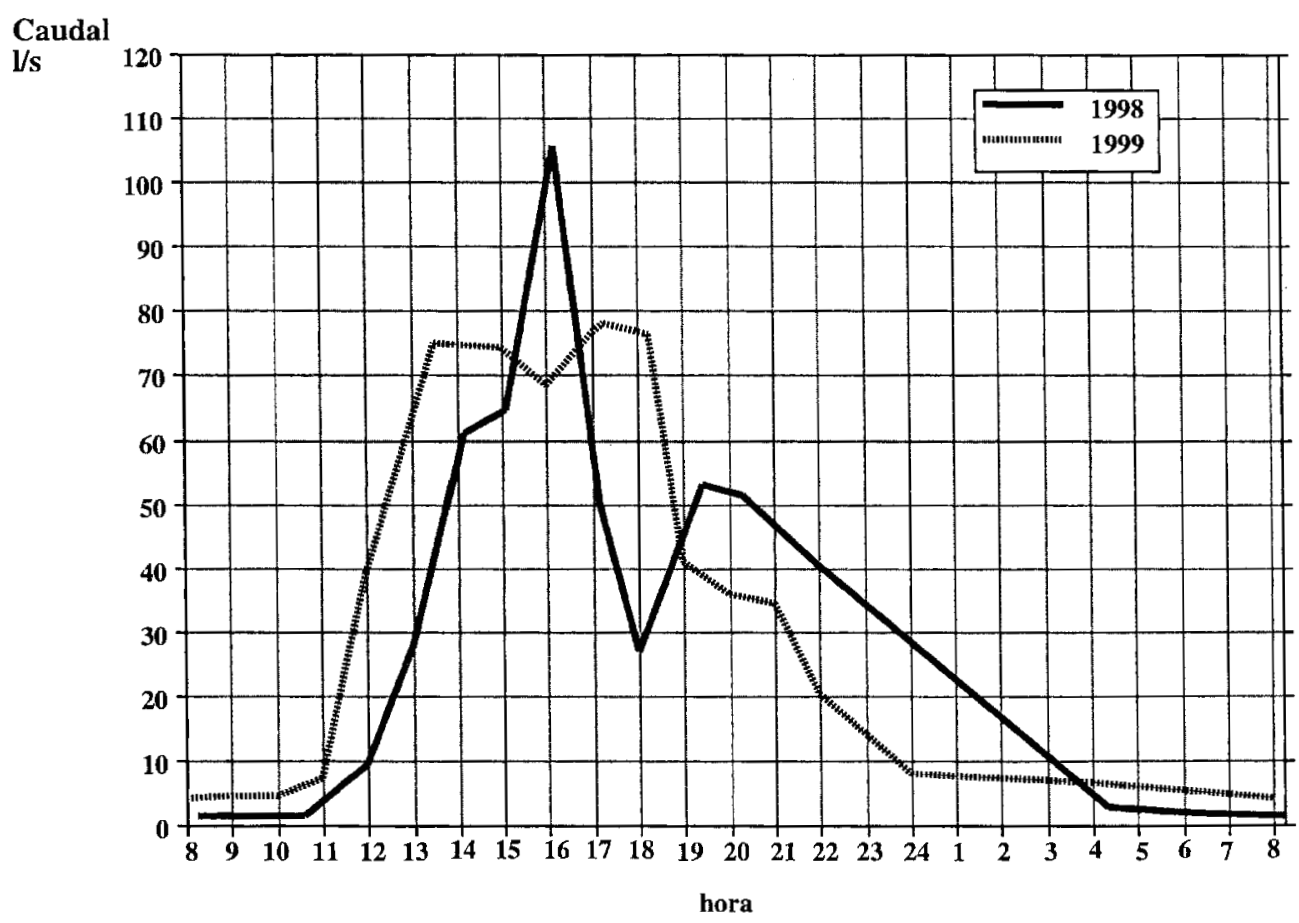

Figura 2. Caudales de fusión en el glaciar del Infierno (4-5 de septiembre de 1998 y 10-11 de septiembre de 1999) 
Sin embargo, la temperatura ambiental fue 1 ó 2 grados más alta que el día precedente, y éste tuvo que ser el factor clave de la mayor fusión.

- El total de caudal de fusión derivado de la ablación glaciar en las 24 horas (45 de septiembre), calculado a partir de las mediciones realizadas, se estima en 2.303.507 litros, con lo que el caudal medio en esas 24 horas fue de $26,66 \mathrm{l} / \mathrm{s}$. Supone el $0,33 \%$ del caudal medio del río Gállego en el aforo del embalse de Búbal para esta época del año (estimado en $8 \mathrm{~m}^{3} / \mathrm{s}$ a partir de datos de Marín, 1989). El caudal específico, teniendo en cuenta que la superficie del glaciar es de 9,6 hectáreas, asciende a $277,71 / \mathrm{s} / \mathrm{km}^{2}$, valor diez veces superior al caudal específico del Gállego en Búbal en agosto-septiembre: $27,58 \mathrm{l} / \mathrm{s} / \mathrm{km}^{2}$.

Sobre la fusión glaciar en 1999 (10-11 de deptiembre), varios aspectos, que exponemos a continuación, llaman la atención en relación con los datos del año anterior. Las figuras 2,3 y 4 , junto a la tabla 2 , sirven de apoyo gráfico y numérico a estas explicaciones.

Temp.

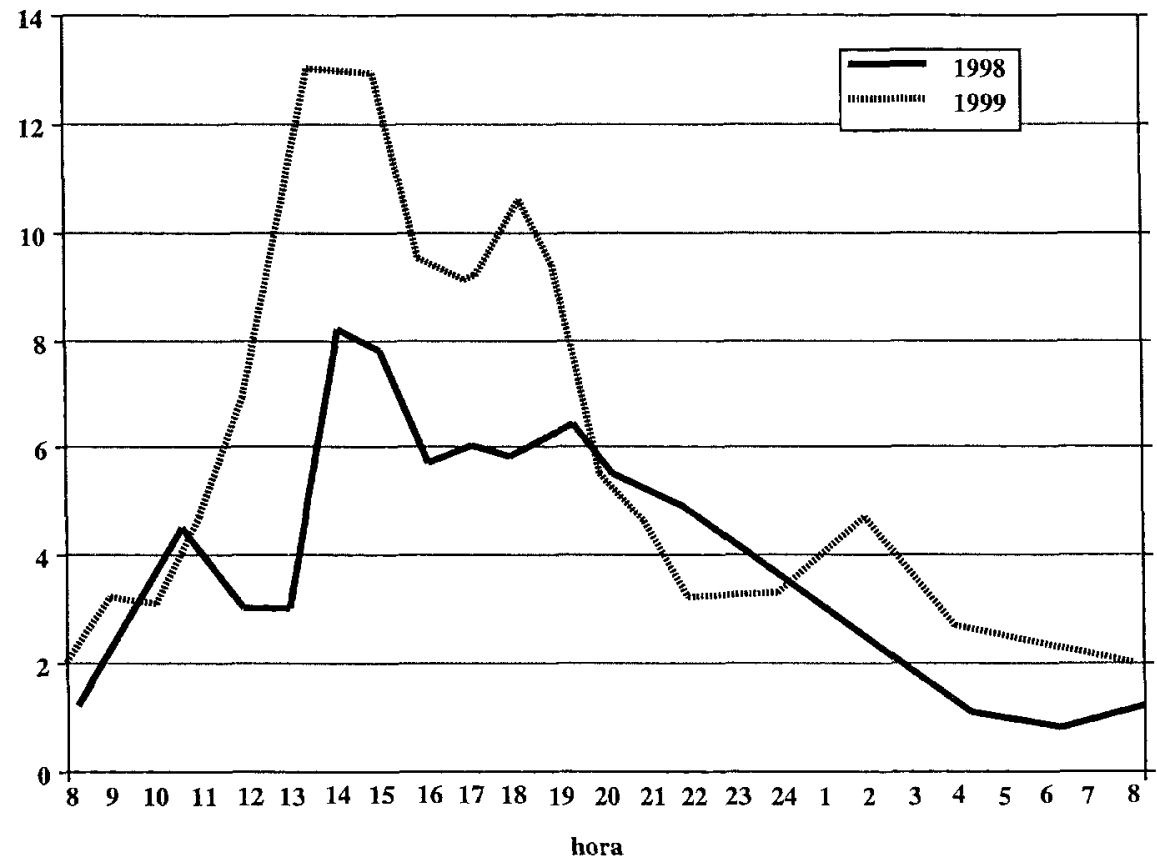

Figura 3. Temperatura ambiental en la base del glaciar del Infierno (4-5 de septiembre de 1998 y 10-11 de septiembre de 1999) 


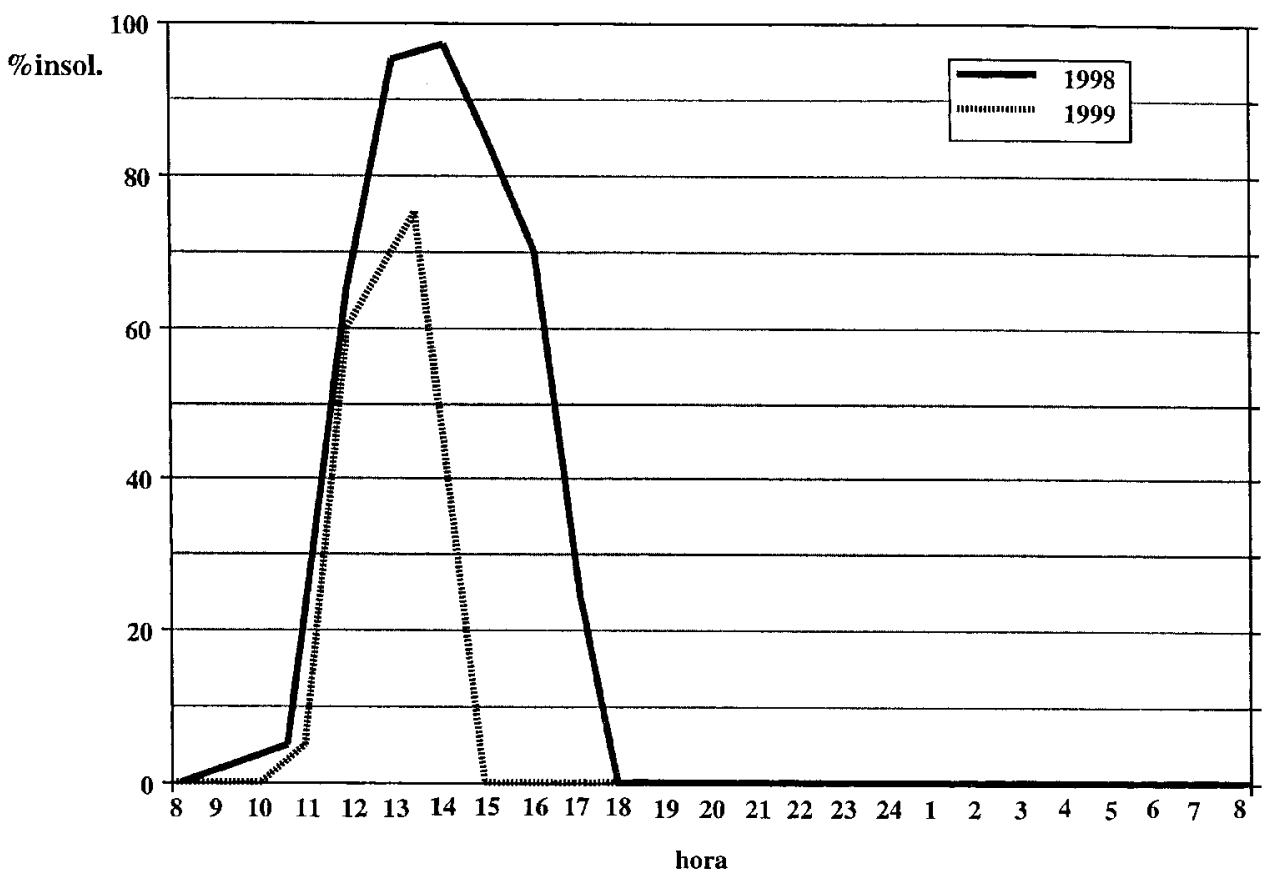

Figura 4. Insolación sobre porcentaje de superficie del glaciar de Infiernos (4-5 de septiembre de 1998 y 10-11 de septiembre de 1999)

- No se registra un pico marcado de máximo caudal sino un periodo prolongado de aguas altas de unas 5 horas (desde las 13:30 h hasta las 18:15 h).

- El máximo caudal parece registrarse alrededor de las 17 h, si bien coincide con el inicio y conclusión de las medidas en los dos días sucesivos, por lo que no podemos asegurar este dato. Si así fuera, significa que en las observaciones de 1999 el máximo de fusión se registró aproximadamente una hora más tarde que en 1998.

- El periodo de aguas bajas se prolonga con valores muy similares desde las 24 $\mathrm{h}$ a las $11 \mathrm{~h}$, registrándose el mínimo a las $8 \mathrm{~h}$. Teniendo en cuenta la considerable longitud de los periodos de aguas altas y aguas bajas, las curvas de ascenso y descenso de caudal son muy pronunciadas, por lo que se trata de dos procesos rápidos en el tiempo.

- El cociente entre el caudal máximo y el mínimo arroja un valor de 19,74, muy inferior al valor de 84,4 registrado en 1998. En efecto, el caudal máximo (78,2 
Tabla 1. Toma de datos hídricos en el torrente colector del Glaciar del Infierno (4-5 de septiembre de 1998)

\begin{tabular}{|lllr|}
\hline Hora & Temperat. del aire ${ }^{\circ} \mathrm{C}$ & Observaciones & Caudal //s \\
\hline $19: 25$ & 6,4 & Cubierto, precipitación insignificante, insolación glaciar 0\% & 53,00 \\
$20: 20$ & 5,5 & Cubierto, precipitación insignificante, insolación glaciar 0\% & 51,30 \\
$21: 52$ & 4,9 & Niebla y lluvia débil & 41,03 \\
$4: 20$ & 1,1 & Poco nuboso, viento & 2,87 \\
$6: 25$ & 0,8 & Poco nuboso, viento & 1,64 \\
$8: 14$ & 1,2 & Poco nuboso, viento & 1,25 \\
$10: 38$ & 4,5 & Poco nuboso, viento, comienza insolación sobre el glaciar & 1,54 \\
$12: 00$ & 3,0 & Despejado, viento, 65\% del glaciar insolado & 9,45 \\
$13: 00$ & 3,0 & Despejado, viento, 95\% del glaciar insolado & 27,92 \\
$14: 10$ & 8,2 & Despejado, viento, 97\% del glaciar insolado & 60,78 \\
$15: 05$ & 7,8 & Despejado, viento flojo, 85\% del glaciar insolado & 64,50 \\
$16: 12$ & 5,7 & Despejado, viento, 70\% del glaciar insolado & 105,51 \\
$17: 10$ & 6,0 & Despejado, viento flojo, 25\% del glaciar insolado & 50,00 \\
$18: 00$ & 5,8 & Despejado, viento flojo, insolación sobre el glaciar 0\% & 26,92 \\
& & Caudal medio 24 horas & 26,66 \\
& &
\end{tabular}

Nota: la hora que indicamos en todo el trabajo es la oficial. La solar corresponde a dos horas menos

Tabla 2. Toma de datos hidricos en el torrente colector del Glaciar del Infierno (10-11 de septiembre de 1999)

\begin{tabular}{|lrlr|}
\hline Hora & Temperat. del aire ${ }^{\circ} \mathrm{C}$ & Observaciones & Caudal l/s \\
\hline $17: 15$ & 9,2 & Despejado al $50 \%$, insolación glaciar $0 \%$ & 78,22 \\
$18: 15$ & 10,6 & Despejado al 40\%, insolación glaciar $0 \%$ & 76,50 \\
$19: 00$ & 9,4 & Despejado al 45\%, insolación glaciar 0\% & 40,94 \\
$20: 00$ & 5,5 & Despejado al 70\%, insolación glaciar 0\% & 35,66 \\
$21: 00$ & 4,6 & Noche & 34,37 \\
$22: 00$ & 3,2 & Noche & 20,45 \\
$24: 00$ & 3,3 & Noche & 7,86 \\
$02: 00$ & 4,7 & Noche & 7,11 \\
$04: 00$ & 2,7 & Noche & 6,64 \\
$08: 00$ & 2,0 & Despejado al 100\%, insolación glaciar 0\% & 3,96 \\
$09: 00$ & 3,2 & Despejado al 100\%, insolación glaciar 0\% & 4,58 \\
$10: 00$ & 3,1 & Despejado al 95\%, insolación glaciar 0\% & 4,60 \\
$11: 00$ & 4,7 & Despejado al 90\%, insolación glaciar 5\% & 7,30 \\
$12: 00$ & 6,9 & Despejado al 70\%, insolación glaciar 60\% & 39,10 \\
$13: 30$ & 13 & Despejado al 40\%, insolación glaciar 75\% & 75,06 \\
$15: 00$ & 12,9 & Despejado al 15\%, insolación glaciar 0\% & 74,35 \\
$16: 00$ & 9,5 & Despejado al 15\%, insolación glaciar 0\% & 68,29 \\
$17: 00$ & 9,1 & Cubierto al 100\%, insolación glaciar 0\% & 76,55 \\
& & Caudal medio 24 horas & 29,13 \\
\hline
\end{tabular}

Nota: la hora que indicamos en todo el trabajo es la oficial. La solar corresponde a dos horas menos 
l/s) es inferior al del año anterior (representa un 74\%); y el mínimo $(3,96 \mathrm{l} / \mathrm{s}$ ) es más de tres veces superior al de 1998. Es decir, podemos indicar que el régimen de fusión de 1999 es menos extremado.

- La comparación con el gráfico de temperaturas no resulta tan clara como en el año anterior. No existe respuesta hidrológica, por ejemplo, a un puntual aumento térmico a las $2 \mathrm{~h}$ de la madrugada. Quizás si nos movemos en temperaturas inferiores a $5^{\circ} \mathrm{C}$, como es el caso, la respuesta en fusión a un incremento térmico débil sea inapreciable.

- Por lo que respecta a los valores totales de fusión en las 24 horas, podemos estimar un caudal medio de 29,13 l/s, un caudal específico de $303,4 \mathrm{l} / \mathrm{s} / \mathrm{km}^{2}$ y un total de 2.516 .953 litros; es decir, 213.446 litros más que en el año anterior, lo que representa un incremento del 9,27\%. Este hecho, que puede ser debido a varias causas, debemos relacionarlo en gran parte con la temperatura ambiente: la media del 4-5 de septiembre de 1998 fue de $3,8^{\circ} \mathrm{C}$, mientras que la del 10-11 de septiembre de 1999 ascendió a 5,5 C.

Los valores de fusión obtenidos en los veranos de 1998 y 1999, acabados de presentar, pueden ser comparados con los medidos en el Pirineo francés hace más de 70 años. En efecto, en 1929, la Administration des Eaux et Forêts (del Ministerio de Agricultura francés), encargó un estudio a M. Joseph Devaux, publicado en la revista Études Glaciologiques, que muy posiblemente sea la primera aproximación al fenómeno de la fusión glaciar en los Pirineos. Las medidas, llevadas a cabo en el macizo de Vignemale, dan una idea del agua originada en un glaciar: a finales de septiembre, con temperaturas medias levemente superiores a los $0^{\circ} \mathrm{C}$, el glaciar d'Ossue (el mayor del complejo glaciado del Vignemale) proporciona alrededor de $30 \mathrm{~m}^{3}$ por hectárea y día con tiempo nuboso (reducida insolación), y unos $300 \mathrm{~m}^{3}$ por hectárea y día con cielo despejado (elevada insolación). Devaux ya apuntaba entonces varios factores que condicionarían la fusión glaciar: a) la altitud, orientación y pendiente de la masa de hielo; b) la estación o época del año; c) las circunstancias meteorológicas imperantes: nubosidad, temperatura, etc. (Devaux, 1934).

En nuestro caso, los valores de fusión glaciar medidos el 4-5 de septiembre de 1998 proporcionan unos valores de $239,9 \mathrm{~m}^{3} / \mathrm{ha} /$ día, mientras que los del 10-11 del mismo mes en 1999 suponen 262,1 $\mathrm{m}^{3} / \mathrm{ha} /$ día. Ambos son plenamente comparables a los medidos por Devaux. Estos datos nos permite realizar un ejercicio de cálculo de la pérdida de espesor equivalente de la capa de hielo. Los $239,9 \mathrm{~m}^{3} / \mathrm{ha} /$ día de fusión del 4-5 de septiembre de 1998 equivalen a 23,99 litros $/ \mathrm{m}^{2} /$ día, es decir, a una columna de $23,99 \mathrm{~mm} /$ día de agua. Suponiendo una densidad del hielo de 0,9 esos $23,99 \mathrm{~mm}$. de agua equivalen a $26,65 \mathrm{~mm}$ de espesor en hielo. Respecto a los $262,1 \mathrm{~m}^{3} / \mathrm{ha} / \mathrm{dí}$ del 10-11 de septiembre de 1999, y aplicando el mismo razonamiento, equivalen a $29,12 \mathrm{~mm}$ de espesor en hielo. 
Lógicamente, tales valores de denudación glaciar, muy elevados, se deben producir sólo unos pocos días al año, a finales de agosto y principios de septiembre, precisamente en las épocas de nuestras mediciones, cuando el glaciar está en su mayor parte libre de la capa de nieve protectora y las temperaturas aún son altas. En fechas anteriores y posteriores, la pérdida de espesor afectará más a la cobertura nival que al propio cuerpo de hielo.

\section{Mediciones de retroceso en el Glaciar del Infierno}

En la campaña estival de 1998 instalamos en este glaciar varios puntos de control en enclaves rocosos estables inmediatos al cuerpo de hielo, desde los que poder medir con precisión en años sucesivos la evolución de dicho glaciar. Tras el primer año de control podemos afirmar con rotundidad que la evolución regresiva de la masa de hielo es no sólo evidente, sino de gran magnitud. Entre el 5 de septiembre de 1998 y el 11 de septiembre de 1999 hemos registrado las siguientes variaciones:

- El frente glaciar ha retrocedido $8,5 \mathrm{~m}$ en su punto de máximo avance, además de haberse adelgazado sensiblemente.

- El retroceso lateral que hemos podido medir, en el tercio inferior del glaciar, es de $5 \mathrm{~m}$.

- En 1999 hemos podido constatar que todo el sector inferior del glaciar (lengua terminal) puede ser considerado glaciar negro, cubierto íntegramente por clastos, en una longitud de $54 \mathrm{~m}$. Por contra, en 1998 sólo había una guirnalda de glaciar negro en la margen derecha del glaciar. Insistimos en que esos $54 \mathrm{~m}$. se refieren a toda la superficie del hielo cubierta por piedras, ya que desde ese límite hacia arriba continúan abundantes guirnaldas de clastos, pero entre ellas se instala el hielo blanco.

- Estimamos una pérdida de potencia (grosor), en el tercio inferior, de $1 \mathrm{~m}$ aproximadamente.

- Los bedières que surcan el tercio inferior del glaciar son más numerosos en 1999 que en 1998, además de mayores: más anchos y más profundos. En varios casos su nivel basal (talweg) puede estar cercano al sustrato rocoso del cuerpo de hielo. Caso de alcanzarse éste, el glaciar perdería su actual masividad, quedando fragmentado en varios lentejones de hielo incomunicados.

Se trata, en todos los casos, de valores de retroceso muy rápidos, especialmente significativos si tenemos en cuenta que se refieren a un glaciar de pequeñas dimen- 
siones, lo cual permite augurar una pronta desparición del mismo, si no varían las actuales condiciones climáticas.

\section{Conclusiones}

Los glaciares pirenaicos se encuentran sometidos a un proceso de rápida regresión consecuencia de unas condiciones climáticas poco favorables para su persistencia. El estudio de los volúmenes de fusión glaciar y de sus ritmos diarios ha sido realizado en el glaciar del Infierno, masa helada con forma de triángulo invertido o embudo que facilita la concentración de la mayor parte de sus caudales de fusión en un único colector. Las mediciones se han efectuado a finales de dos veranos sucesivos, para evitar en lo posible el efecto de la fusión nival sobre los caudales y limitarnos a la fusión propiamente glaciar. De los datos obtenidos cabe destacar las muy importantes variaciones de caudales día-noche y la magnitud de los volúmenes diurnos, que permiten disponer de un argumento más para entender la importante regresión glaciar actual. Esta regresión ha podido ser constatada fehacientemente mediante medidas efectuadas en varios puntos de control instalados en el Glaciar del Infierno: en sólo un año se aprecian importantes pérdidas de potencia y superficie. Por otra parte, se ha podido comprobar la muy directa respuesta del glaciar, en cuanto a sus ritmos de fusión, a las variaciones térmicas registradas en los días de medición entre 1998 y 1999. Así, aun cuando los volúmenes de fusión son globalmente mayores en este último año, los ritmos son menos extremados que en 1998. Los valores obtenidos ambos años son muy corcordantes con los medidos hacia 1930 en el glaciar d'Ossue, en el Pirineo francés.

\section{Notas}

1 Los datos horarios indicados en este trabajo se refieren a la hora oficial. La solar, en estas fechas veraniegas, corresponde a dos horas menos. Hacemos esta observación pará evitar, en su caso, posibles confusiones derivadas de la comparación de resultados entre diferentes trabajos que tratan de los ritmos de fusión glaciar.

2 Este plan de mediciones se trastocó levemente en la campaña de 1998, pues una precipitación en forma de lluvia entre las $22 \mathrm{~h}$. del día cuatro de septiembre y las $2 \mathrm{~h}$. del día cinco aconsejó no realizar mediciones en ese periodo, y esperar algo más de dos horas tras el final del evento para eliminar su efecto en el caudal.

\section{Agradecimientos}

Este trabajo se enmarca en los siguientes proyectos de investigación: 
- Referencia 247-50, de la Universidad de Zaragoza (España): “Catálogo actualizado, cartografía geomorfológica y bases para el seguimiento comparado de la dinámica de los glaciares altoaragoneses..

- Referencia P08/98, de la Diputación General de Aragón (España): "Bases para el seguimiento comparado de la dinámica de los glaciares altoaragoneses según análisis climáticos, hidrológicos y geomorfológicos».

- Referencia P043/99 de la Diputación General de Aragón (España): "Seguimiento de la dinámica de los glaciares altoaragoneses según análisis climáticos, hidrológicos y geomorfológicosn.

Queremos mostrar nuestro agradecimiento a Miguel Sánchez Fabre, Anabel Moreno, Ahmed Elmoujahid y José Antonio Cuchí por su colaboración.

\section{Bibliografía}

Arenillas, M., Cantarino, I., Martínez Costa, R., Martínez de Pisón, E. y Pedrero, A. (1992): El control de los glaciares actuales en el Programa ERHIN. En MOPT La nieve en las cordilleras españolas. Programa ERHIN, año 1990/91, Madrid, p. 215-227.

Arenillas, M., Ferrer, C., Martínez de Pisón, E., Martínez, R., Navarro, J. y Yagüe, J. (2000): Surveillance generale des glaciers espagnols. Leur etat critique à la fin du XXème siècle. En SHF Section de GlaciologieNivologie. Symposium 1-2 mars 2000, Grenoble (Actes du Symposium). Societé Hydrotechnique de France.

Arenillas, M., Martínez Costa, R., Martínez de Pisón, E. y Navarro, J. (1998): Los glaciares actuales y la nieve en el Pirineo español. En Gómez Ortiz, A. y Pérez Alberti, A. Las buellas glaciares de las montañas españolas, Servicio de Publicacions da Universidade de Santiago de Compostela, p. 329-346.

Bocquet, G. y Thomas, A. (1989): Mesures hydrologiques et climatologiques en haute montagne. Les handicaps du milieu. Revue de Géographie Alpine, LXXVI (1-23), p. 95-104.
Cancer, L. (1995): Ecogeografia de los paisajes del Alto Gállego. Consejo de Protección de la Naturaleza de Aragón, Zaragoza.

Cancer, L., Lampre, F., Ollero, A., Del Valle, J., Sánchez, M. y Cuchí, J.A. (2001): La fusion glaciaire sur les glaciers de l'Infiernos et de la Maladeta (Pyrénées, Espagne) aux dernières années du vingtième siècle: quelques resultats dans le domaine de l'environnement. En SHF Section de Glaciologie-Nivologie. Symposium 15-16 mars 2001, Grenoble (Actes du Symposium). Societé Hydrotechnique de France.

Cancer, L., Lampre, F., Ollero, A., Del Valle, J., Sánchez, M. y Cuchí, J.A. (en prensa): La fusion glaciaire sur les glaciers de l'Infiernos et de la Maladeta (Pyrénées, Espagne) entre 1998 et 2000: quelques resultats dans le domaine de l'environnement. Houille Blanche. Societé Hydrotechnique de France, Paris.

Chueca, J. y Lampre, F. (1994): Los glaciares altoaragoneses. Instituto de Estudios Altoaragoneses, Diputación de Huesca, Huesca. 
Chueca, J., Peña, J.L., Lampre, F, García Ruiz, J.M. y Martí Bono, C.E. (1998): Los glaciares del Pirineo Aragonés: estudio de su evolución y extensión actual. Universidad de Zaragoza, Zaragoza.

Devaux, M.J. (1934): Étude des radiations solaires sur les glaciers et des glaces souterraines dans les Pyrénées (Études de 1929). Études Glaciologiques, Tome VII, 5 : 218-238.

Lampre, F. (1998): Estudio geomorfológico de Ballibierna (Macizo de la Maladeta, Pirineo Aragonés): modelado glacial y periglacial. Consejo de Protección de la Naturaleza de Aragón, Zaragoza.

Lampre, F., Cancer, L. y Ollero, A. (1999): Ritmo diario de los caudales de fusión en el glaciar de Infiernos (Pirineo aragonés). En Pallí, L. y Roqué, C. (Eds.) Avances en el estudio del Cuaternario español (secuencias, indicadores paleoambientales y evolución de procesos), AEQUAUniversitat de Girona, Girona, p. 173-180.

Marín, J.M. (1989): Balance hídrico e hidrológico de la cuenca alta del río Gállego. Geographicalia, 26 p. 175-181, Zaragoza.

Martínez de Pisón, E. y Arenillas, M. (1988): Los glaciares actuales del Pirineo Español.
En MOPU: La nieve en el Pirineo Español, p. 29-98. Madrid.

MOPU (1988): La nieve en el Pirineo Español. Ministerio de Obras Públicas y Urbanismo, Madrid.

Ollero, A., del Valle, J., Cancer, L. y Lampre, F. (2000): Análisis comparativo de los caudales de fusión en los glaciares de Infiernos y Maladeta (Pirineo Aragonés). Lurralde, 23, p. 115-123.

René, P. (2001): Les Glaciers des Pyrénées Françaises: campagne d'observations de septembre 2000. Dans SHF Section de Glaciologie-Nivologie. Symposium 15-16 mars 2001, Grenoble (Actes du Symposium). Societé Hydrotechnique de France.

Serrano, E. y Agudo, C. (1988): La deglaciación del circo de los Ibones Azules. Estudio glaciomorfológico. Cuaternario $y$ Geomorfología, 2, p. 115-123.

Sugden, D. y John, B.S. (1976): Glaciers and landscape. A geomorpbological approach. Arnold.

Vivian, R. y Zumstein, J. (1973): Hydrologie sous-glaciaire au glacier d'Argentière (Mont Blanc, France). Symposium on the bydrology of glaciers, Cambridge, 9-13 Sept. 1969, Int. Ass. Scient. Hydrol. 95, p. 53-64. 